is essential to deliver patient-centred PEOLC. There is little in-depth research on the collaboration between general practice and hospice services and the variety of models and processes that currently exist.

Aims To investigate GP experiences and perceptions of hospice care and the facilitators and limitations to SPC referral and shared care management.

Method A GP interview study across a CCG. Semi-structured interviews were performed and a framework methodology employed.

Results Twelve GPs participated. They described how use of hospice services is influenced by their own experience; practice expertise; perceptions of patients' needs; and relationships with the hospice. Key themes were GP factors; perceived accessibility and responsiveness of hospice services; patient and family factors, which affect referral decision-making; and effective professional relationships. GPs viewed urgent referrals or referrals for patients from ethnic minorities as most challenging. They also struggled to identify the right time to refer patients with non-malignant diagnoses. GPs uniformly preferred personal communication with clinical nurse specialists and relied on stable professional relationships suggesting that care was disrupted when CNS cover was lacking.

Conclusions This study gives weight to the challenge of partnership working across teams of different palliative care professionals, and illustrates how professional relationships are central to effective collaboration, referral and patient management. We recommend further research into robust collaborative working and an evaluation of equity of access to services particularly for ethnic minorities. We also suggest the need for further development of contemporaneous electronic patient notes and GP education. These with stable service delivery are vital to planning future models of care and proactive service delivery.

\section{0-18 GSF AND HOSPICE PARTNERSHIP WORKING AS REGIONAL CENTRES DELIVERING GSF CARE HOMES TRAINING}

Keri Thomas, Clare Fuller, Denise Douglas, Annabel Foulger, Anne Keating. Gold Standards Framework Centre C.I.C. Shrewsbury, UK

10.1136/bmjspcare-2017-hospice.18

Background Care homes, where a fifth of the population die, are crucial providers of end-of-life care (EOLC), for the elderly with frailty and dementia, but many require further EOLC training. The Gold Standards Framework Care Homes (GSFCH) Training Programme provides a more proactive, person-centred, systematic approach adopted within care homes, enabling better care with fewer avoidable admissions and hospitals deaths, and more dying where they choose. The GSF Centre developed partnerships with several hospices as Regional Centres as part of the national cascade of the GSFCH Programme.

Aims To describe the development of GSF Regional Centre to cascade training to facilitate embedded sustainable proactive end-of-life care for residents in line with their wishes and preferences.

Methods Twelve regional centres were recruited through an application process and a process of staged train-the-trainers developed ensuring quality assurance of the GSF Care Homes Programme delivery to a wider local audience, usually involving four to six workshops over several months. Training included earlier identification, clinical assessment, advance care planning discussions and planning living and dying well. Intrinsic comparative evaluation data is collated to evaluate the impact. Many homes then progress to Accreditation with the recognised GSF Quality Hallmark Award and many more are re-accredited, demonstrating sustainability.

Results Results from the Regional Centres delivery of the GSF $\mathrm{CH}$ Programme will be presented including numbers trained, attainment of standards, and impact factors. The benefits of a collaborative approach are further discussed showing the benefit to hospices of this shared approach.

Conclusion Partnership working between GSF Centre, hospice regional centres and care homes facilitates spread of this successful programme, benefits to hospices with increased uptake of other training and benefits to care homes enabling more residents receiving better EOLC and more dying where they choose. Further areas of development will be discussed.

\section{0-19 EVALUATION OF THE 'HOSPICE IN YOUR CARE HOME' PROJECT}

1,2Katherine Froggatt, 1,2Nancy Preston, 1,2Rachael Eastham, 1,2Garuth Chalfont, ${ }^{1,2}$ Debbie Dempsey. International Observatory on End of Life Care, Lancaster University, Lancaster, UK; ${ }^{2}$ Wigan and Leigh Hospice, Wigan, UK

\subsection{6/bmjspcare-2017-hospice. 19}

Background There is limited evidence on the impact of multicomponent interventions to support the delivery of palliative care in care homes. In 2015, one hospice established an innovative 'Hospice in Your Care Home' team using a number of interventions: role modelling and working alongside staff, responses to urgent referrals, advance care planning and training courses. This project has been externally evaluated.

Aims

- To evaluate the process and outcomes of the 'Hospice in Your Care Home' initiative, with specific reference to hospital admissions and end of life care practices.

- To ascertain the costs of delivering the project.

- To identify facilitators and barriers to the implementation of the initiative.

Methods The responsive evaluation comprised:

- an analysis of secondary service provision data

- focus group interviews with care home managers $(n=7)$, care home staff $(n=11)$ and the project team $(n=6)$

- preliminary analysis of cost (time and finance).

Secondary data was analysed using descriptive and inferential statistics. Framework analysis structured the qualitative data analysis.

Results Nine care homes participated with one facility leaving and one joining part way through. Hospital admissions were significantly reduced by $25 \%(\mathrm{p}=0.01)$, between 2015 and 2016. Resident status meetings were conducted in each facility, with 4479 residents discussed at 217 meetings. Staff described increased confidence in their ability to care for residents with palliative care needs. To establish this project three stages of implementation were identified: initiation, assimilation, and 'everyday' running. The project was facilitated by the hospice team's flexibility and supportive approach. Reported barriers reflected the ongoing challenges regarding staffing levels and release of staff to attend training. 
Conclusions This hospice model has demonstrated significant reductions in hospital admissions and increased confidence of care home staff in end of life practices. It has the potential to be expanded in other localities.

\section{0-20 WHEN 999 GETS CALLED AT THE END OF LIFE: EQUIPPING AMBULANCE CLINICIANS TO PROMOTE A DIGNIFIED DEATH (HOSPICES AND AMBULANCE SERVICE WORKING TOGETHER)}

${ }^{1}$ Claire Nicell, ${ }^{2}$ Richard Smith, ${ }^{3}$ James Hill, ${ }^{4}$ Sarah Stead. ${ }^{1}$ The Hospice of St Francis, Berkhamsted, UK; ${ }^{2}$ East of England Ambulance Service NHS Trust, Watford, UK; ${ }^{3}$ East of England Ambulance Service NHS Trust, Chelmsford, UK; ${ }^{4}$ East of England Ambulance Service NHS Trust, Bedford, UK

\subsection{6/bmispcare-2017-hospice.20}

Background End of life care is an emergent aspect of ambulance clinicians' work. They are often called to an emergency or crisis in an individual's last days of life and have to make complex decisions with limited information. However they receive limited formal palliative care education. Two hospices were approached by a local paramedic to see if they could help address this educational need.

Aim of the Collaborative Work

- For ambulance clinicians to gain confidence in recognising end of life needs of patients and their families and delivering appropriate care.

- For hospice professionals to understand the role and abilities of ambulance clinicians in delivering end of life care.

What have we achieved?

- Two hospices and their local Ambulance NHS Trust worked collaboratively to devise a one-day workshop for paramedics to explore palliative and end of life care.

- The workshop included communication skills, symptom control, recognising dying, promoting patient wishes and accessing local palliative care services.

- Feedback from 70+clinicians was good and their confidence scores increased

- 'Helps make a grey area of our work more understood.'

- Two paramedics completed the European Certificate in Essential Palliative Care and are recognised locally as palliative care champions within their work in the community and control room.

- Links established with local university; end of life care sessions delivered to third-year paramedic students and palliative care student placements arranged.

Additional Benefits

- Increased mutual understanding of practitioners' roles informing practice and education.

- Paramedics accessing ongoing communication skills training.

- Paramedics delivering teaching to hospice staff highlighting end of life care challenges.

- Improved communication between ambulance crews and local hospices.

- Joint hospice and ambulance end of life care Facebook discussion delivered as part of the Ambulance's service End of Life Care Awareness month

- Joint end of life care strategy working.

\section{Lessons learnt in engagement and governance}

\section{0-21 DRIVING CULTURE CHANGE: CROSS-ORGANISATIONAL ENGAGEMENT WITH PEOPLE WITH PERSONAL EXPERIENCE}

Andrea Dechamps. St Wilfrid's Hospice, Eastbourne, UK

10.1136/bmjspcare-2017-hospice.21

Background User involvement in hospices, whilst acknowledged as important, can be viewed as particularly difficult with people nearing end of life, can become the sole domain of a particular worker/expert (e.g., the palliative care social worker) and can lack overall buy-in from across organisations. Two years ago our hospice disbanded our main vehicle for user involvement, the Patient Forum, in their own judgement by now weary and unoriginal. Under a PPE (People with Personal Experience) governance stream we embarked on an open-ended, ambitious drive innovating engagement with PPE. Aims

- To work towards cross-organisation engagement with PPE, through multiple time-limited PPE initiatives led by volunteers, clinical and non-clinical staff at all levels and across settings.

- To instil a sense of curiosity and develop competence amongst all staff and volunteers regarding PPE involvement.

- Ultimately, for PPE involvement to significantly influence service delivery and development across settings.

Methods

- Definition of PPE revised to include our patients, families, customers, public, community, staff and volunteers.

- Key performance target set at 20 PPE initiatives for 2016/17 demonstrated senior commitment.

- Support given to project leaders, with focus on '(almost) anything goes'

- Initiatives mapped by project leaders against business priorities, CQC Key Lines of Enquiry, Quality Improvement Priorities and themes from complaints.

Results Twenty diverse initiatives delivered 2016/2017. Two showcasing events. Demonstrable changes to service delivery across the organisation resulting from above plus culture change amongst staff and volunteers.

'I am just beginning to understand what PPE involvement means - it matters!'

'Involving families in how we design $\mathrm{xx}$, designing $\mathrm{xx}$ in collaboration, has made a real difference.'

Conclusion The above describes the first significant stage of a new approach to PPE involvement. We already have demonstrable evidence of quality improvement as a result plus culture change. We believe the described approach to be innovative and have potential for duplication in other settings.

\section{0-22 CQC REQUIRES IMPROVEMENT TO OUTSTANDING IN ONE YEAR: A HOSPICE JOURNEY}

Kerry Harrison. Heart of Kent Hospice, Aylesford, UK

10.1136/bmjspcare-2017-hospice.22 